\title{
III.
}

\section{Senkungsabscess unterhalb der Pars mastoidea und Retropharyngealabscess infolge von acuter eitriger Media.}

\author{
(Aus der Ohrenabtbeilung der Kgl. chirurg. Universitätspoliklinik \\ zu München.)
}

Ton

Dr. Hang.

Privatdocent in Hünchen.

\begin{abstract}
O. L., 17 Jahre alt, ein im allgemeinen ziemlich kräftig entwickeiter junger Mann, bisher immer ohrgesund, acquirirte im Anschluss an starken Nasenrachenkatarrh Anfangs Mai 1895 eine acute eitrige Mittelohrentzündung, die am 4. Tage ihres Bestandes zur Spontanperforation des linken Trommelfelles fübrte. Der zugerufene Arzt behandelte die Erkrankung mit Ausspritzungen von Borwasser und Insufflationen von Borpulver. Der Eiterfluss soll die erste Zeit sehr reichlich gewesen sein, vom 8 . Tage ab ungefähr aber schnell abgenommen und beinabe ganz aufgehört haben, gleichzeitig seien aber die verschwunden gewesenen Schmerzen im Ohre wieder von Neuem aufgetreten, und etliche Tage darauf soll Schmerzhaftigkeit in der' Warzenfortsatzgegend mit Schwellung derselben bemerkbar geworden sein. Das Hören auf der kranken Seite war erlosehen, der Allgemeinzustand infolge der Schmerzen und des erneuten Fiebers ein ziemlich schlechter. Als die Schmerzen immer stärker wurden, ebenso die Schwellung, so dass Patient den Kopf nicht mehr drehen konnte, zudem sich noch Kopfschmerzen intensiver Natur und Schwindel hinzugesellten, wurde Patient an mich verwiesen.
\end{abstract}

Status. Am 28. Mai 1895. Im linken Gehörgang wenig halbeingetrockneter Eiter. Gehörgang stark verengt infolge allgemeiner Schwellung, hauptsächlichaber durch Senkung der hinteren Gehörgangswand. Das Trommelfell kann nur mit Mühe zu Gesicht gebracht werden; es ist bleigrau und vorgebuchtet; streckenweise mit weissen Epitbelfetzen belegt; nach unten, von der Mittellinie etwas nach hinten, ist eine kleine Lücke zu erlennen, die auf einem Granulationszapfen ;sitat. Die Präauriculargegend geschwellt und schmerzhaft, so dass alle Kieferbewegungen nur unter grössten Schmerzen ausgeführt werden können. Den Hals kann Patient nicht mehr drehen, er hält inn wie bei Torticollis berubergebeugt, eine Folge der Schwelling und Infiltration der Mastoidealgegend. Die Infiltration selbst ist ziemlich bart, derb, von bräunlichviolettrother Farbe und erstreckt sich von der Schläfenbeinschuppe beginnend, die ganze seitliche Halsregion einnehmend und auf die Hinterhauptpartie diffus iubergehend, bis beinahe auf die Clavicula fort. Die ganze schwellung ist diffus, Fluctuation ist nur ganz undeutlich unterhalb des Warzenfortsatzes nach hinten zu fühlen. Die Empfindlichkeit der Partie ist sehr gross. Im Kieferwinkel sind geschwollene, schmerzhafte Drüsenpackete eben noch zu constatiren. Das Allgemeinbefinden ist ein schlechtes; Patient ist ziemlich comatös, reagirt nur weníg Temperatur $39,9^{\circ}$. Puls $96^{\circ}-100^{\circ}$. Seit 2 Tagen sollen uberdies Schluck- und Schlingbeschwerden bestehen. Die 
Inspection des Halses kann nur mit Mühe erzwungen werden; die linke Gaumenhälfte und die Tonsillengegend zum Pharynx hin erscheinen etwas hyperämischer als rechts; sonst findet sich nichts. Eigentliche Schwellung ist nicht vorbanden, ebenso wenig irgendwelcher Belag; es war auch in der ganzen vorhergehenden Zeit keine Angina vorhanden gewesen; erst in den letzten 2 Tagen waren Schluckbeschwerden aufgetreten.

Es warde nun sofort die Paracentese des Trommelfelles ausgeführt, zugleich aber der ansgedehnte operative Eingriff in Aussicht gestellt als wahrscheinlich unumgänglich nothwendig. Bei der Paracentese, die nur mühsam wegen der Enge des Gebörganges ausgefübrt werden konnte, entleerte sich thatsächlich eine ziemliche Menge angestauten Eiters, und füblte Patient sich im Ohre selbst freier, soweit man bei ihm durch Fragen zum Ziele kommen konnte. Da Einpinselungen mit Jod und Eisblase schon vorher ohne Erfolg versucht worden waren, so wurde weiterhin abgesehen hiervon.

Am năchsten Tage war zwar ziemlich viel Eiter aus dem Ohre ausgeflossen, aber das Allgemeinbefinden und die Schmerzhaftigkeit in der Hinterohrgegend war noch die gleiche, eher noch etwas vermehrt. Temp. Abds. $39,0^{\circ}$. Puls $92-96$.

Es wurde deshalb den darauffolgenden Tag, 30. Mai 1896 die Operation ausgeführt, deren Einzelheiten hier blos kurz angedeutet werden sollen. Weichtheile allenthalben derb speckig infiltrirt; starke parenchymatöse Blutung. Periost dunkel verfärbt, aber adhärent. Corticalis nicht durchbroshen, nicht mächtig. Nach Wegnahme derselben durch den grossen Amputationshohlmeissel $(1,2 \mathrm{Cm}$. breit) kommt ein sebr pneumatischer Warzenfortsatz zum Vorschein, dessen fast sämmtliche Höhlen mit Eiter und Granulationen vollgepfropft sind. Antrum, etwa gut erbsengross, enthält blos Eiter. An der Spitzenzelle, die ebenfalls sehr ausgebildet ist, findet sich ebenfalls dicker Eiter, und von ihm aus gelangt man mittelst der Sonde in eine nach hinten und unten von der Spitze des Processus gelegene grössere Tasche. Es wurde deshalb eine zweite Incision in der Richtung des Verlaufes des Kopfniekers gesetzt und langsam praeparando, so weit das bei der starken Infiltration möglich war - die Infiltration war hier über $2^{1 / 2}-3 \mathrm{Cm}$. stark - bis die Fascie und der hintere Rand des Kopfnickers freiliegen, vorgegangen, und es gelang, dem hier ganz in dieser Tiefe angesammelten Eiter Abfluss zu verschaffen. Stumpfe Erweiterung der Lücke.

Trotz dieser den augenblicklichen Symptomen entsprechenden Eingriffe, trotz scheinbaren Rückganges der Schwellung in der Hinterohrgegend, fiel das Fieber nicht nur nicht, abgesehen von einer kleinen Remission, die ebenso gut blos durch den Blutverlust bedingt gewesen sein kann, wie durch die Eiterentleerung, sondern stieg noch um etliche Zehntel; der Patient wird eher noch mehr apathisch, soporös, das Schlingen erweist sich als sehr erschwert und schmerzbaft, zudem gesellt sich beginnende Athemnoth. Die linke Rachenhalfte, vom harten Gaumen beginnend, ist, nachdem sie sich schon die Tage vorher als injicirt und leichter geschwellt gezeigt batte, jetzt enorm diffus geschwollen, so dass Gaumenbogen, Tonsille und Retropharynx eine über eigrosse pralle Vorwölbung bilden. Temp. $39,8^{\circ}$. Puls 112 , sehr dünn.

Es bandelt sich also offenbar am einen pharyngealen oder retropharyngealen Abscess, und es muss deshalb, 2 Tage nach der Mastoidealoperation, gesucht werden, dem Halseiter ebenfalls Abfluss zu verschaffen. Die erste Incision an der prominenten Partie des weichen Gaumens, die scheinbar das Gefübl der Fluctuation hat, blieb resultatlos; ebenso die zweite in der peritonsillären Schwellung ausgeführte. Auch die dritte, die an einer sackartig nach hinten und unten von der Tonsille gelegenen Partie, welche noch gegen das Gaumensegel, resp. zur Gegend des Levatorwulstes nach oben zu auslief, gemacht wurde, schien anfänglich resultatlos verlaufen zu wollen; erst bei stumpfer Erweiterung mit dem Listerzängelchen, nachdem in dem enorm geschwollenen Gewebe über $21 / 2 \mathrm{Cm}$. in die Tiefe vorgegangen worden war, entleerte sich endlich dicker, gelber Eiter, (etwa ein Kaffeelöffel voll reinen Eiters, das Blut abgerechnet). Sowohl in dem aus dem Senkungsabscesse der pars mastoidea als in dem aus dem Retropharyngealabscess entleerten Eiter fand sich Bacillus pneumoniae. 
Offenbar war erst dieser pharyngeale Eingriff der entscheidende gewesen, denn von da $a b$ besserte sich das Allgemeinbefinden zusehends in raschem Tempo, nachdem das Fieber am Tage der Incisionen auf $38,6^{\circ}$ abgefallen war. Der comatöse Zustand fing an, ebenfalls gleichzeitig zurïckzugehen, die Athempassage war sofort frei geworden, die Schlingbeschwerden erheblich zurückgetreten. Am Tage nach der Incision, in der Nacht hatte Patient noch eine relativ erhebliche Menge Eiters nachträglich per os entleert, war die Morgentemperatur $37,6^{\circ}$. Die Geschwulst im Halse gemindert, ebenso aber auch die Schwellung aussen in der Gegend des Kieferwinkels und der Submaxillardrüse. Athmung ruhig und tief. Abends Temp. 37,60. Puls 72.

Der Verlauf war nun weiterhin zunächst bezüglich des inneren Halsabscesses eine rasche, völlige Zurückbildung, so dass Nahrungsaufnahme wieder bei gutem Appetit reichlich erfolgen konnte.

Die Infiltration und Schwellung der äusseren Halspartien hinunter bis zur Clavicula und der Mastoidea-Occipitalregion ging bedentend langsamer zurück, wenn schon bei dem ersten Verbandwechsel (am 4. Tag nach der Operation) eine nicht unwesentliche Minderung zu constatiren war. Jedenfalls konnte Patient jetzt den Kopf schon viel freier beinahe ohne Schmerzempfindung drehen. Aber erst nach dem 3. Verbandwechsel, wobei sich jedesmal sowohl der obere in der Mastoidwunde steckende Gazestreifen als insbesondere der untere in die Senkungshöhle eingeführte als stark mit Eiter imprägnirt erwiesen (also am 12. Tage nach der Operation), konnte die Infiltration als im Wesentlichen gelöst betrachtet werden; eine gewisse Derbheit und Schwellung war aber immer noch vorhanden. 'Von jetzt ab verringerte sich auch die Secretion aus dem oberen Wundkanale, wăhrend die untere Incision nach dem 4. Verband gar kein Secret mehr absonderte und sich trotz Tamponade bald schloss.

Vom 5. Verbandwechsel ab war der Meatus trocken; Trommelfell geschlossen; Hörfähigkeit nun wieder 150 Ctm. Flüstersprache. Definitive Heilung ám Ende der 6. Woche, auch der Mastoidöfnung. Hörvermögen annähernd wieder normal.

\section{Epikritische Betrachtungen.}

Es bietet dieser Fall in zweierlei Hinsicht Interessantes dar, einmal beziuglich des Durchtrittes des Eiters unterhalb des Warzenfortsatzes und dann hauptsächlich aber wegen der Abscedirung im retrotonsillaren Gewebe. Wir haben es also im Ganzen mit atypischen Senkungen zu thun.

Was das erstere, die Senkung unterhalb des eigentlichen Warzenfortsatzgebildes selbst anbelangt, so können wir uns bierüber sehr kurz fassen: es ist einer der Fälle von sogen. Bezoldscher Mastoiditis, die jetzt oft genug schon beschrieben worden sind. Auch hier konnte wieder, wie das wohl bei den meisten derartigen Mastoiditen der Fall gewesen ist, das eigenthümlich grosszellige und stark pneumatisehe Geftige des W arzenfortsatzes und insbesondere auch die starke Entwicklung der terminalen Spitzenzelle constatirt werden; in dieser anatomisch präformirten Anordnung liegt ja theilweise der Grund nicht nur zum leichten Mitergriffenwerden der Pars mastoidea, sondern specieller auch noch für diesen tiefen Durchbruch des Eiters unter die Ansätze der seitlichen Halsmuskeln. Bemerkenswerth wäre in unserem 
Falle nur die ausserordentliche Ausdebnung der harten Infiltration bis weit auf die Hinterhanptgegend.

Interessanter und wichtiger ist das Auftreten der retrotonsillaren Abscedirung im Gefolge der acnten Mittelohrentzündung fast gleichzeitig mit der Mastoiditis. Es sind ja schon einzelne derartige Fälle beschrieben worden, aber sie sind nicht immer in der günstigen Weise verlaufen, wie z. B. im Falle Knapp's, der allerdings etwas sehr spät als Senkungsabscess erkannt wurde, bei der Section. Man könnte ja vielleicht einwenden, hier habe es sich gar nicht um eine Complication vom Ohre aus gehandelt, sondern lediglich um eine accidentelle, zufällig gleichzeitige, phlegmonöse Angina.

Allein ich glaube kaum, dass wir hier auf diese reflectiren dürfen, wenn es auch das einfachste a priori vielleicht erscheinen möchte. Von einer Halsinfection von aussen ber durch den Mundrachenraum dürften wir wohl also absehen; es war auch während der ganzen Erkrankungszeit niemals ein Belag auf den Tonsillen oder Lacunenentzündung zu beobachten gewesen, ganz abgesehen davon, dass bei dem Patienten die Momente zu einer Infection durch Uebertragung von anderer Seite völlig in Wegfall kamen. Ebenso kann aueh als eventuell ursächlich vermittelndes Moment das Vorhergehen irgend weleher mit Angina sich complicirenden Allgemeininfection ausgeschlossen werden. Erst im Anschlusse an die Erkrankung des Warzenfortsatzgebietes greift die Affection successive auf den Pharynx über. Gleichzeitig oder kurz nachdem sich die von der Pars mastoidea ausgehende Schwellung über den Gehörgang erstreckt, dabei das Gebörgangslumen durch die Schwellung in äusserstem Grade verengt hatte, und von diesem aus wieder über die Fascia parotideo masseterica hinübergekrochen war, entwickelt sich die anfänglich nur stark spannende Infiltration der Rachenpartien, die dann unter Schmerzen im Halse zu der Abscedirnng in der Tiefe der Retrotonsillargegend fübrt. Die gegenüberliegende Halsseite war und blieb immer während des ganzen Krankheitsverlaufes von jedem Anzeichen einer Entziindung absolut frei.

Da nun der massenhaft im ganzen Mittelobr (Pauke und Warzentheile) angesammelte Eiter weder durch den Gehörgang, noch durch die Tube genügenden Abfluss fand, so brach er sich einerseits Bahn unter den Warzenfortsatz, resp. unter die in der Regio Mastoidea ansetzenden Muskelpartien, andererseits aber brach er nach innen und unten von der Trommelhöhle zu durch, indem 
er in dem die Tube umlagernden Gewebe sich zum Levator veli, der ja bekanntlich durch häntige Massen längs der Tube zum Theile befestigt verläuft, hin zog und dort sich concentrirend $\mathrm{zu}$ der Vorwölbung unter dem Bilde der Retropharyngealabscedirung Veranlassung gab. Es ist dabei wohl wahrscheinlich, dass der Semicanalis pro tensore tympani die Rolle eines Vermittlers, eines Leiters der Infection zwischen Mittelohr und Pharynx gespielt hat; auch die Möglichkeit des sofortigen Eindringens in das peritubare Gewebe als solches ist nicht von der Hand zu weisen.

Ich glaube also für diesen Fall als sicher annehmen zu dürfen, dass es sich nicht um nur accidentelle phlegmonöse Abscedirung handelte, sondern um einen retropharyngealen Senkungsabscess vom eitrig erkrankten Mittelohr aus. Einen Stützpunkt für diese Anschauung gewinnen wir des weiteren noch aus der sowohl direct nach der Warzenfortsatzeröffnung als auch der nach der Incision des Halsabscesses vorgenommenen bacteriologisehen Prifung des entleerten Eiters: beide enthielten neben Stceptokokken in der Hauptsache den Pneumobacillus, der auch rein aus beiden Herden cultivirt werden konnte.

Wenn auch diese Art des Verlaufes einer eitrigen Media als eine entschieden seltene, als eine Ausnahme bezeichnet werden muss, so legt dieser Fall von Neuem wieder dem Praktiker dar, dass wir bei jeder acuten eitrigen Mittelohrentzïndung zu jeder Zeit ihres Bestandes die grösste Wachsamkeit handhaben müssen. Sobald iuber irgendwelche stärkere Spannung in der seitlichen Halsgegend, vom Rachenwinkel ans beginnend, geklagt wird, muss eine Inspection und Palpation statthaben. Sehr häufig werden sich diese Erscheinungen, die ja gar nicht selten im Verlaufe von acuter Tympanitis in leichtem Maasse zur Beobachtung gelangen, als der "Tubenschmer" zur baldigen spontanen Rückbildung gelangen, aber wir dürfen nicht vergessen, dass eben unter Umständen eine ganz unvermittelt ohne jede Infectionsgelegenheit auftretende Angina oder ein scheinbar unerklärlicher Retropharyngealabscess seinen Ursprung einer Mittelohreiterung verdanken kann. Auf den Spontandurchbruch, wenn die peritonsilläre phlegmonöse Entzündung gemäss der Allgemein- und Localsymptome zur Eiterung tendirt, zu warten, ist gerade bei diesen Senkungsabscessen sehr gewagt, da sie infolge der tiefen Lage des Eiters eher sich noch mehr nach unten senken werden, als spontan durchbrechen. Allerdings darf man sich durch etliche vergebliche Incisionen nicht von dem vorgesetzten 
Ziele abbringen lassen, insbesondere wird das sehr tiefe, wenn auch zum Theile stumpfe Vorgehen in dieser gewiss nicht ungefährlichen Gegend eine gewisse Vorsicht erfordern. Ein rechtzeitiger Eingriff am rechten Ort wird hier, wie so oft, das Leben des Patienten erhalten können. -

Anhangsweise möchte ich hier kurz noch eines Falles Erwähnung thun, der, ebenfalls als acute Media verlaufend, sich mit dem Bilde einer typischen Torticollis verband. Es handelte sich um einen Studierenden der Medicin, der im Frühjahre 1896 eine acute exsudative Media acquirirte. Es stellten sich bald exquisite Schüttelfröste mit starken nächtlichen Temperatursteigerungen ein, und dazu gesellte sich eine hochgradige Nackensteifigkeit. Ein College sah die ganze Sache für eine Torticollis rheumatica an.

Als sich Patient vorstellte, war das Trommelfell verdickt, aber stark ausgebuchtet, mattroth. Der Warzenfortsatz an der Spitze sehr druckempfindlich, aber kaum leichte Infiltration nachzuweisen. Der Hals ist ganz gegen die kranke Seite zu geneigt und kann nur unter den grössten Schmerzen seitwärts bewegt und gedreht werden. Nach der sofort vorgenommenen Paracentese, die eingedicktes, eitriges Secret entwickelt, verschwinden alle Symptome, sowohl die Torticollis war vom nächsten Tage ab verschwunden, als auch blieben die vorher öfters schon dagewesenen Schüttelfröste aus. Eine Eiterung trat nach der Paracentese nicht ein. 